\title{
Expression of HIF-1 $\alpha$ and VEGF in Skeletal Muscle of Plateau Animals in Response to Hypoxic Stress
}

\author{
H.-C. XIE ${ }^{1,2}$, J.-P. HE ${ }^{1}$, J.-F. ZHU ${ }^{2}$, J.-G. LI ${ }^{1}$ \\ ${ }^{1}$ College of Life Science, Shaanxi Normal University, Xi'an, China, ${ }^{2}$ The Key Laboratory of \\ Education Ministry on Environments and Resources in Tibetan Plateau, Qinghai Normal \\ University, Xining, China
}

Received June 12, 2014

Accepted August 29, 2014

\begin{abstract}
Summary
Hypoxia-inducible factor-1a (HIF-1a) transcriptionally regulates expression of several target genes in protecting tissues against hypoxia. With hypoxic stress, vascular endothelial growth factor (VEGF) is a signal protein produced by cells and further contributes to improvement of vascular functions and restoring the oxygen supply to tissues. In this current study, we first hypothesized that the protein levels of HIF-1a and VEGF are reduced in skeletal muscles of plateau animals [China QinghaiTibetan plateau pikas (ochotona curzoniae)] in response to hypoxia as compared with control animals [normal lowland Sprague-Dawley (SD) rats]. We further hypothesized that HIF-1a plays a role in regulating expression of VEGF in skeletal muscle. Note that HIF-1a and VEGF were determined by using two-site immunoenzymatic assay (ELISA) methods. Our results demonstrated that hypoxic stress induced by exposure of lower $\mathrm{O}_{2}(6 \mathrm{~h})$ significantly increased the levels of HIF-1a and VEGF in the oxidative and glycolytic muscles of SD rats and pikas $(P<0.05$ vs. normoxic conditions). Notably, the increases in HIF-1a and VEGF were significantly less in pikas ( $P<0.05, v s$. SD controls) than in SD rats. In addition, a linear relationship was observed between amplified HIF-1a and VEGF in oxidative muscle $(r=0.76$ and $P<0.01)$ and glycolytic muscle $(r=0.72$ and $P<0.01)$ and inhibiting HIF-1a significantly decreased expression of VEGF induced by hypoxic stress in skeletal muscles $(P<0.05)$. Overall, our findings suggest that (1) responsiveness of HIF-1a and VEGF in skeletal muscles to hypoxic stress is blunted in plateau animals, and (2) HIF-1a has a regulatory effect on VEGF under hypoxic environment.
\end{abstract}

\section{Key words}

HIF-1a • VEGF • Skeletal muscle • Hypoxic stress

\section{Corresponding author}

J.-G. Li, College of Life Science, Shaanxi Normal University, 620 West Chang'an Avenue, Xi'an 710119, China. E-mail: jingangli64@gmail.com

Hypoxia inducible factor-1 (HIF-1) has an important contribution to physiological changes of homeostasis under conditions of oxygen deprivation as well as tissue ischemia. Accumulated subunit HIF-1 $\alpha$ modulates the expression of several target genes in protecting tissues against hypoxia and ischemia (Ceradini et al. 2004, Manalo et al. 2005). In addition, with inadequate oxygen supply and blood flow vascular endothelial growth factor (VEGF) is considered as a signal protein produced by cells and further plays an important role in improving vascular functions and vasculogenesis and thereby leading to restoring the oxygen supply to tissues (Kim and Byzova 2014).

Plateau pikas (ochotona curzoniae) are the predominant species of small native mammals to live on the China Qinghai-Tibetan plateau alpine meadow with an altitude of approximately $3000 \mathrm{~m}$. They are completely acclimatized to the hypoxic environment (Du and $\mathrm{Li} \mathrm{1982).} \mathrm{Also,} \mathrm{as} \mathrm{a} \mathrm{unique} \mathrm{model} \mathrm{of} \mathrm{hypoxic}$ tolerance plateau pikas have been used to study the physiological and ecological adaptive responses to acute or chronic hypoxic stress as well as the underlying cellular and molecular mechanisms related to hypoxic 
environment (Chen et al. 2007).

Skeletal muscle fibers can be characterized by their metabolic processes, namely oxidative and glycolytic muscles, e.g. oxidative fibers primarily use oxidative phosphorylation to generate ATP (Lieber 2002). Also, oxidative fibers contain a large number of myoglobin, an oxygen-binding protein that can store oxygen and speed its delivery to mitochondria within the muscle cell. In contrast, glycolytic muscle has the less levels of myoglobin and relies on glycolysis to supply muscle energy (Lieber 2002). Nonetheless, it is largely unknown how hypoxic environment influences on expression of HIF-1 $\alpha$ and VEGF in those two distinct muscle fibers.

Therefore, in the current study, we examined the protein expression of HIF- $1 \alpha$ and VEGF in the oxidative and glycolytic muscles of plateau pikas and lowland control animals in response to hypoxic stress induced by exposure of lower $\mathrm{O}_{2}$. Moreover, we injected an inhibitor of HIF-1 $\alpha$ [(2-methoxyestradiol (2-MeOE2)] into the hindlimb muscles and then examined the levels of VEGF in skeletal muscle to determine the effects of HIF-1 $\alpha$ on VEGF. We hypothesized that responses of HIF-1 $\alpha$ and VEGF are blunted in skeletal muscles of plateau pikas during hypoxia as compared with lowland control animals. Also, we hypothesized that HIF-1 $\alpha$ can contribute to expression of VEGF in skeletal muscle.

All procedures outlined in this study were in accordance with the Guide for the Care and Use of Laboratory Animals (US National Institute of Health), and approved by the animal Care Committee of this institution. Fifty-one healthy adult male plateau pikas (150-200 g, obtained from the Qinghai Alpine Meadow, $\sim 3000 \mathrm{~m}$ altitude) and sixty-six Sprague-Dawley (SD) rats (from Lab Animal Center of this Institute) were used in this study. Hypoxic stress was performed in a chamber of $70 \times 70 \times 50 \mathrm{~cm}$ ventilated with a mixed gas $\left(\mathrm{O}_{2} / \mathrm{N}_{2} \%\right)$ of $21 \% \quad(\sim$ sea level $)$ as control, and of $10.8 \% \mathrm{O}_{2}$ ( $\sim 5000 \mathrm{~m}$ altitude) as hypoxic stimulator (Chen et al. 2007), which was controlled by oxygen sensor (Innovative Instruments Co.). In additional group of experiments, 2-MeOE2 (10 mg, Selleck Chem. Co.) was injected into the hindlimb muscles (gastrocnemius muscle) of animals with hypoxia. All the animals were kept in the chambers for $6 \mathrm{~h}$, and then decapitated following overdose of isoflurane in order to remove the gastrocnemius muscle of the hindlimb. Note that the deep red and superficial white portions of the gastrocnemius muscle are composed predominantly of oxidative and glycolytic fibers, respectively.
The levels of HIF-1 $\alpha$ (Abcam Co.) and VEGF (R\&D System) were determined using a two-site immunoenzymatic assay (ELISA) according to the provided description and modification. Briefly, polystyrene 96-well microtitel immunoplates were coated with affinity-purified polyclonal rabbit anti-HIF-1 $\alpha$ antibody/mouse anti-VEGF antibody. Parallel wells were coated with purified rabbit/mouse IgG for evaluation of nonspecific signal. After overnight incubation at room temperature and $2 \mathrm{~h}$ of incubation with the coating buffer containing $50 \mathrm{mM}$ carbonate buffer (pH 9.5) in $2 \% \mathrm{BSA}$, plate were washed with $50 \mathrm{mM}$ Tris- $\mathrm{HCl}$. After extensive washing, the diluted samples and the HIF-1 $\alpha /$ VEGF standard solutions were distributed in each plate and left at room temperature overnight. The plates were then washed and incubated with anti-HIF-1 $\alpha /$ anti-VEGFgalactosidase per well. Then, the plates were washed and incubated with substrate solution. After an incubation of $2 \mathrm{~h}$ at $37^{\circ} \mathrm{C}$, the optical density was measured using an ELISA reader (Dynatech).

All data were analyzed using a two-way repeatedmeasure analysis of variance due to two interventions (normoxic and hypoxic conditions) in two groups of animals. Values are presented as means \pm standard error (SE). For all analyses, differences were considered significant at $P<0.05$. All statistical analyses were performed by using SPSS for Windows version 15.0.

First, in a subset of group we examined the basal levels of HIF-1 $\alpha$ and VEGF in oxidative and glycolytic muscle of SD control rats $(n=6)$ and pikas $(n=6)$. No significant differences were observed in the baseline of HIF- $1 \alpha$ and VEGF between two groups. In SD rats, HIF- $1 \alpha$ was $1.76 \pm 0.20 \mathrm{ng} / \mathrm{ml}$ in oxidative muscle and $2.51 \pm 0.22 \mathrm{ng} / \mathrm{ml}$ in glycolytic muscle; and in pikas, HIF-1 $\alpha$ was $1.68 \pm 0.16 \mathrm{ng} / \mathrm{ml}(P>0.05 v s$. SD control) in oxidative muscle and $1.97 \pm 0.31 \mathrm{ng} / \mathrm{ml}(P>0.05$ vs. SD control) in glycolytic muscle. In addition, VEGF was $25.65 \pm 2.33 \mathrm{pg} / \mathrm{ml}$ in oxidative muscle and $27.62 \pm 3.12$ $\mathrm{pg} / \mathrm{ml}$ in glycolytic muscle of SD control rats; and $23.45 \pm 2.81 \mathrm{pg} / \mathrm{ml}(P>0.05$ vs. SD control $)$ in oxidative muscle and $24.89 \pm 3.20 \mathrm{pg} / \mathrm{ml}(P>0.05$ vs. SD control) in glycolytic muscle of pikas.

Moreover, as noted in Figure 1 (top panel) that under normoxic condition HIF-1 $\alpha$ levels of both oxidative and glycolytic muscle in pikas tend to be lower than those in SD rats, but there were no significant differences in HIF-1 $\alpha$ between the two groups. Nevertheless, Figure 1 (top panel) shows that hypoxic stress significantly increased the levels of HIF-1 $\alpha$ in the 
oxidative and glycolytic muscles of SD rats and pikas as compared with control normoxic conditions. In oxidative muscle, $\%$ increase of HIF-1 $\alpha$ was $79 \pm 5 \%$ in SD control rats $(n=20)$ and $49 \pm 4 \%$ in pikas $(n=15, P<0.05$ vs. SD rats). In glycolytic muscle, $\%$ increase of HIF-1 $\alpha$ was $98 \pm 6 \%$ in SD control rats $(\mathrm{n}=20)$ and $65 \pm 5 \%$ in pikas ( $\mathrm{n}=15, P<0.05 v s$. SD rats).

Figure 1 (bottom panel) demonstrates that hypoxic stress significantly increased expression of VEGF in the oxidative and glycolytic muscles of SD rats and pikas as compared with control conditions. In oxidative muscle, \% increase of VEGF was $62 \pm 4 \%$ in SD rats $(n=20)$ and $28 \pm 3 \%$ in pikas $(n=15, P<0.05$ vs. SD rats). In glycolytic muscle, $\%$ increase of VEGF was $82 \pm 6 \%$ in SD rats $(n=20)$ and $36 \pm 3 \%$ in pikas $(n=15$, $P<0.05$ vs. SD rats).
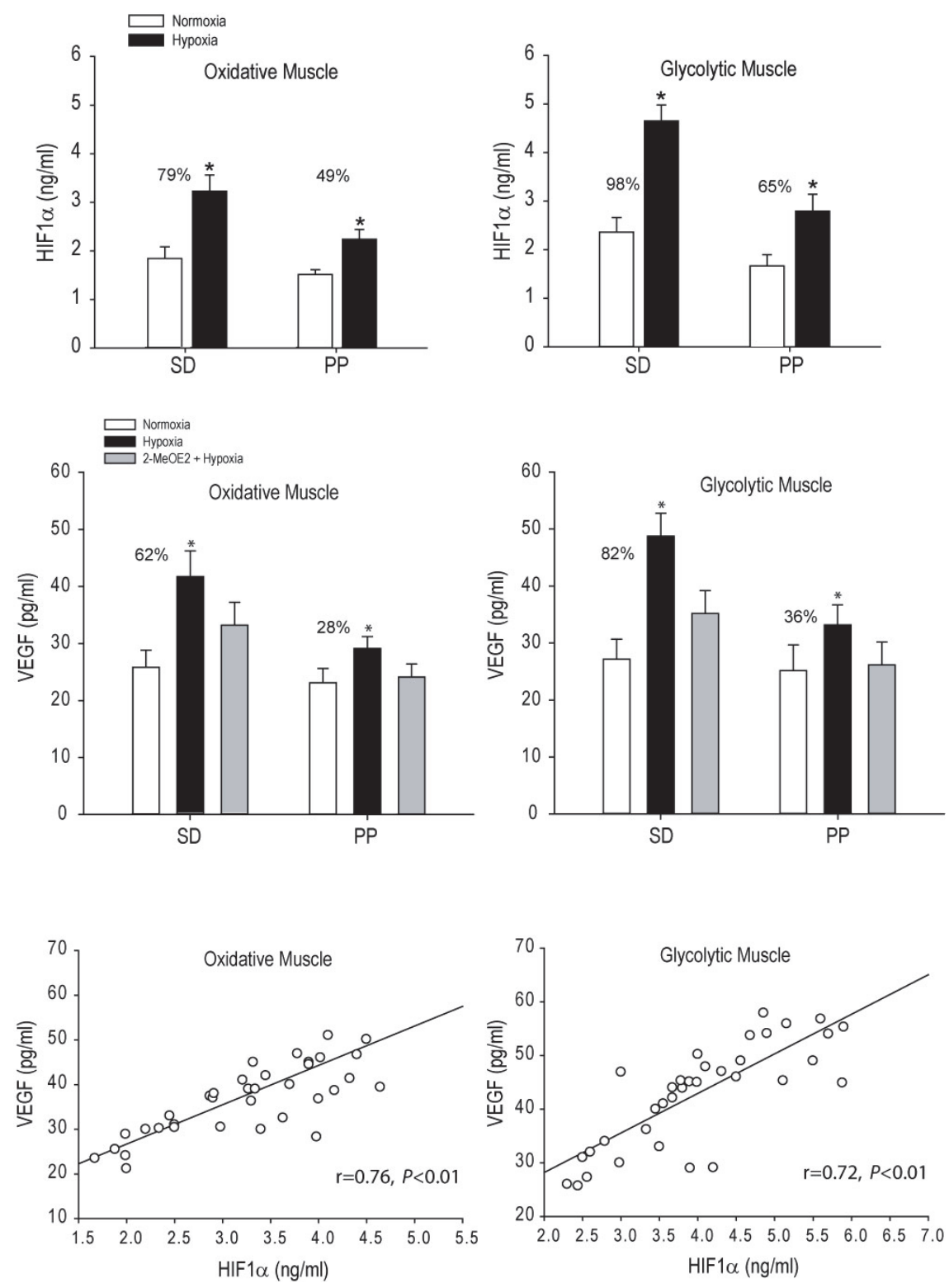

Fig. 1. Hypoxic stress significantly increased the levels of HIF-1a (top panel) and VEGF (bottom panel) in oxidative and glycolytic muscles of SD rats ( $n=20$ for each condition) and pikas ( $n=15$ for each condition). Also, $\%$ increases of HIF-1a and VEGF were attenuated to a greater degree in pikas than that in SD rats. * $P<0.05, v S$. normoxia. In addition, inhibiting HIF-1a by injecting 2-MeOE2 into the hindlimb muscles significantly attenuated expression of VEGF (bottom panel). $* P<0.05$ vs. normxic and hypoxic conditions with 2-MeOE2. SD: SpragueDawley rats; PP: Plateau Pikas.
In addition, in Figure 2 a linear relationship analysis shows that there was a close relation between responses of HIF-1 $\alpha$ and VEGF to hypoxia in both oxidative muscle $(\mathrm{r}=0.76$ and $P<0.01)$ and glycolytic muscle ( $\mathrm{r}=0.72$ and $P<0.01)$. Also, Figure 1 (bottom panel) demonstrates that inhibiting HIF-1 $\alpha$ by injecting 2-MeOE2 into the hindlimb muscles $(n=20$ in SD group and $n=15$ in pika group) significantly attenuated expression of VEGF induced by hypoxic stimulation compared with hypoxic groups $(P<0.05)$. Note that we have also examined the levels of HIF-1 $\alpha$ after 2-MeOE2 in order to confirm its effectiveness in this experiment. HIF-1 $\alpha$ response to hypoxia was $3.26 \pm 0.36 \mathrm{ng} / \mathrm{ml} \quad(P<0.05$ vs. injecton of $2-\mathrm{MeOE} 2)$ in oxidative muscle and $4.52 \pm 0.56 \mathrm{ng} / \mathrm{ml}$ 
$(P<0.05$ vs. injection of 2-MeOE2) in glycolytic muscle of $\mathrm{SD}$ rats $(\mathrm{n}=12)$ without injection of 2-MeOE2; and $1.85 \pm 0.22 \mathrm{ng} / \mathrm{ml}$ in oxidative muscle and $2.62 \pm 0.32 \mathrm{ng} / \mathrm{ml}$ in glycolytic muscle after 2-MeOE2 $(\mathrm{n}=12)$.

Skeletal muscles are made of different fibers, namely oxidative and glycolytic muscle fibers, according to the relative involvement of energy metabolisms (Lieber 2002). HIF-1 $\alpha$ has been considered as a critical sensor of cellular oxygen engaged in energy metabolisms (Ceradini et al. 2004, Manalo et al. 2005). In this study, we examined protein expression of the regulatory HIF- $1 \alpha$ in oxidative and glycolytic muscles of the hindlimb. As reported previously (Pisani and Dechesne 2005), we have observed a higher level of HIF-1 $\alpha$ expression in predominantly glycolytic muscles than in predominantly oxidative muscle under normoxic conditions although under normoxic condition no significant differences were observed in HIF-1 $\alpha$ levels of both oxidative and glycolytic muscle between pikas and SD rats. Moreover, response of HIF-1 $\alpha$ was greater in glycolytic muscles than in oxidative muscle during hypoxic stress. Importantly, results of our study demonstrated that the protein levels of HIF-1 $\alpha$ in response to hypoxic stress were impaired in oxidative and glycolytic muscles of plateau pikas compared with lowland animals.

Under normoxic conditions, variations in HIF-1 $\alpha$ content according to skeletal muscle fiber types are related to the myoglobin content. Oxidative fiber muscles with a high level of myoglobin have a low HIF-1 $\alpha$ content (Lin et al. 2002). Likewise, glycolytic fibers expressing a low level of myoglobin (Vogt et al. 2001) have a high HIF-1 $\alpha$ content. A prior study (Grange et al. 2001) has suggested that an $\mathrm{O}_{2}$ regulation system is present mainly on the basis of myoglobin in oxidative fibers and on an HIF-1 complex in glycolytic fibers. Nevertheless, in our study diminished responsiveness of HIF-1 $\alpha$ was observed in both oxidative and glycolytic muscles of plateau animals, indicating that a balance between myoglobin and HIF-1 is likely altered in skeletal muscle when the tissues are under hypoxic environment.

Also, HIF-1 $\alpha$ is engaged in many cellular pathways to play a functional role, including glucose uptake and carbohydrate metabolism involved by hexokinase 1 and 2, lactate dehydrogenase A, pyruvate kinase, etc. (Semenza 2001). In plateau animals, metabolic mechanisms are adjusted to meet hypoxic environment (Chen et al. 2007).

In addition, our results demonstrated that response of VEGF was increased to hypoxic stress in both oxidative and glycolytic muscles of control and plateau animals. Furthermore, a linear relationship analysis suggests that there is a close relation between responses of HIF-1 $\alpha$ and VEGF. Our study further showed that the levels of amplified VEGF in muscles induced by hypoxic stress were attenuated when an inhibitor of HIF-1 $\alpha$ was previously administered into the hindlimb muscles. This indicates that under hypoxic stress HIF-1 $\alpha$ also plays a role in regulating expression of skeletal muscle VEGF, an important contributor to vascular functions and vasculogenesis as oxygen supply and blood flow directed to various tissues are inadequate.

It should be noted that a close relation was observed between HIF-1 $\alpha$ and VEGF in the skeletal muscles in response to hypoxic stress by using a linear relationship analysis. This indicates a possibility that there is a correlation of HIF- $1 \alpha$ and VEGF. Additional study to determine the effects of VEGF inhibitor on HIF-1 $\alpha$ and VEGF would clarify this issue.

In summary, our data suggest that (1) responsiveness of HIF-1 $\alpha$ and VEGF in both oxidative and glycolytic muscles is blunted in plateau animals under hypoxic stress as compared with lowland animals, and (2) HIF-1 $\alpha$ has a regulatory effect on expression of VEGF in skeletal muscle tissue. Findings of our study have an implication to use Qinghai-Tibetan plateau pika to study physiological and ecological adaptive responses to acute or chronic hypoxic stress in humans who living at high altitude and/or who live at a normal sea level but suffer from hypoxic stressors.

\section{Conflict of Interest}

There is no conflict of interest.

\section{Acknowledgements}

This study was supported by grants from China Ministry of Education \#Z2012096 and \#2013-Z-753.

\section{References}

CERADINI DJ, KULKARNI AR, CALLAGHAN MJ, TEPPER OM, BASTIDAS N, KLEINMAN ME, CAPLA JM, GALIANO RD, LEVINE JP, GURTNER GC: Progenitor cell trafficking is regulated by hypoxic gradients through HIF-1 induction of SDF-1. Nat Med 10: 858-864, 2004. 
CHEN X-Q, WANG S-J, DU J-Z, CHEN X-C: Diversities in hepatic HIF-1, IGF-I/IGFBP-1, LDH/ICD, and their mRNA expressions induced by $\mathrm{CoCl} 2$ in Qinghai-Tibetan plateau mammals and sea level mice. Am J Physiol Regul Integr Comp Physiol 292: R516-R526, 2007.

DU JZ, LI QF: Effects of simulated hypoxic acclimation on organism, organ and hematology in Ochotona curzoniae and rats. Acta Theriologica Sinica 2: 472-477, 1982.

GRANGE RW, MEESON A, CHIN E, LAU KS, STULL JT, SHELTON JM, WILLIAMS RS, GARRY DJ: Functional and molecular adaptations in skeletal muscle of myoglobin-mutant mice. Am J Physiol Cell Physiol 281: C1487-C1494, 2001.

KIM Y-W, BYZOVA TV: Oxidative stress in angiogenesis and vascular disease. Blood 123: 625-631, 2014.

LIEBER RL: Skeletal muscle structure, function, and plasticity: Wolters Kluwer Health. Lippincott Williams Wilkins (ed.), Baltimore, Maryland USA, 2002, pp 116-200.

LIN J, WU H, TARR PT, ZHANG CY, WU Z, BOSS O, MICHAEL LF, PUIGSERVER P, ISOTANI E, OLSON EN: Transcriptional coactivator PGC-1a drives the formation of slow-twitch muscle fibres. Nature 418: 797-801, 2002.

MANALO DJ, ROWAN A, LAVOIE T, NATARAJAN L, KELLY BD, YE SQ, GARCIA JG, SEMENZA GL: Transcriptional regulation of vascular endothelial cell responses to hypoxia by HIF-1. Blood 105: 659-669, 2005.

PISANI DF, DECHESNE CA: Skeletal muscle HIF-1alpha expression is dependent on muscle fiber type. J Gen Physiol 126: $173-178,2005$.

SEMENZA GL: Hypoxia-inducible factor 1: oxygen homeostasis and disease pathophysiology. Trends Mol Med 7: 345-350, 2001.

VOGT M, PUNTSCHART A, GEISER J, ZULEGER C, BILLETER R, HOPPELER H: Molecular adaptations in human skeletal muscle to endurance training under simulated hypoxic conditions. J Appl Physiol 91: 173-182, 2001. 